\title{
Group Audio Application with Flash Multicast Streaming Based on RTMFP
}

\author{
Linghong Xue, Fuan Wen, Chunmei Fan \\ Beijing Key Laboratory of Network System and Network \\ Culture \\ School of Network Education \\ Beijing University of Posts and Telecommunications \\ Beijing, China
}

\begin{abstract}
In order to improve the user experience and reduce the operators' cost, Real-Time Rich Internet Application (RIA) has become more and more important compared with other Web Applications. Real-time RIA combines the Multimedia and Real-time experience in the client side, which provides the live collaboration ability to the application and improves the user experience dramatically. This paper illustrates the Flash P2P mechanism including Multicast and Directed Routing in Flash Player 10.1 based on RTMFP (Real-Time Media Flow Protocol). RTMFP is a new communication protocol introduced since Adobe Flash Player 10. Its low latency, endto-end peering capability, security and scalability make it very suitable for developing Real-Time RIA. In addition, we apply this technology to a Group Audio application in order to present the workflow.
\end{abstract}

Keywords-Multicast Streaming, RTMFP, Flash P2P, RealTime RIA

\section{INTRODUCTION}

In recent years, with the rapid development of Rich Internet Application (RIA), many Web Applications supported by Flash, AJAX or Silverlight etc have been deployed everywhere in the Internet. Only using RIA, there is already little improvement to the UX (User Experience). Nowadays, Real-Time feature has attracted more and more IT companies and will become a new growth factor to the UX. Google Instant is a remarkable example using this feature. So Real-Time UX should represent the next generation of Web applications.

Earlier versions of Flash Player use Real-Time Messaging Protocol (RTMP) and require Adobe Flash Media Server (FMS) or Red5 Media Server for interactive collaboration applications or audio/video streaming. While RTMP is an excellent choice for streaming media, shared objects, or remoting, it has limited ability of meeting realtime requirements of interactive audio and video communications.

Adobe Flash Player began to support Real-Time Media Flow Protocol (RTMFP) since version 10. The first generation of RTMFP in Flash Player 10.0 did not support application-level multicast, and media was always sourced from the publishing peer. The second generation of RTMFP began to support groups in Flash Player 10.1 and reduced the load of the source publisher.

\author{
Jun Wang, Xiao Wang \\ Beijing Key Laboratory of Network System and Network \\ Culture \\ School of Network Education \\ Beijing University of Posts and Telecommunications \\ Beijing, China
}

There are few papers introducing this powerful feature in Flash Player, although Flash Player 10.1 has been released more than a year. This paper will dive into the lightweight $\mathrm{P} 2 \mathrm{P}$ solution that does not require a server. What you need is the IP to connect to and the ability to use UDP for communication is also something we have not seen before. Then this paper will explain some important concepts that the Flash P2P API support. At last, there is a Flash P2P group audio demo to demonstrate the performance of the new feature powered by RTMFP.

\section{Multicast In FLash Player 10.1}

Multicast is one of the features of Flash Player 10.1 and it enables you to distribute Net Streams across the peer-topeer mesh. It can be audio, video or even pure data stream (AMF3). With this feature, we can not only support for the end-to-end $\mathrm{P} 2 \mathrm{P}$ connections, which is enough for 2 peer communication but establish more complex connections [6].

\section{A Difference between Unicast and Multicast [6]}

1) Unicast

Unicast simply delivers streams from a server to some clients. For this purpose you can use Flash Media Server and TCP protocols such as RTMP or HTTP protocol. Unicast also costs you a lot of resources. For example, $1 \mathrm{Mbps}$ stream delivered to 1000 clients means 1 GB upstream from server, so CPU demanding and network transit is also huge.

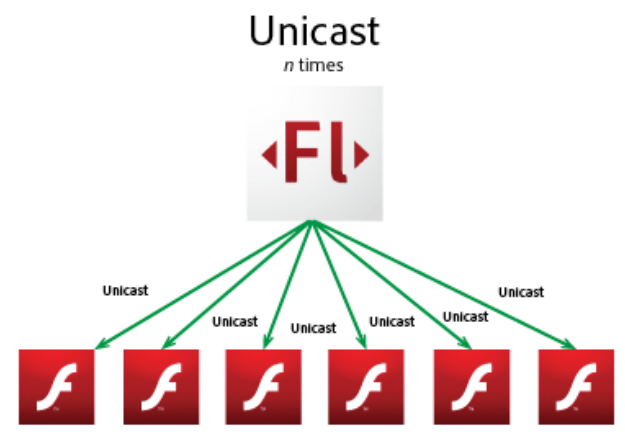

Figure 1. Structure of Flash Unicast

2) Application level Multicast [7] 
Multicast is a highly optimized method to deliver highquality video to a very large audience with virtually no impact on the server as demand increases.

Application level Multicast which is also called P2P Multicast is an optimized stream distribution among peers. Application-level multicast can be used as a one-to-many broadcast or used to support multi way communication between one-to-one or few-to-few scenarios. Application multicast will significantly reduce bandwidth for both public and private network applications.

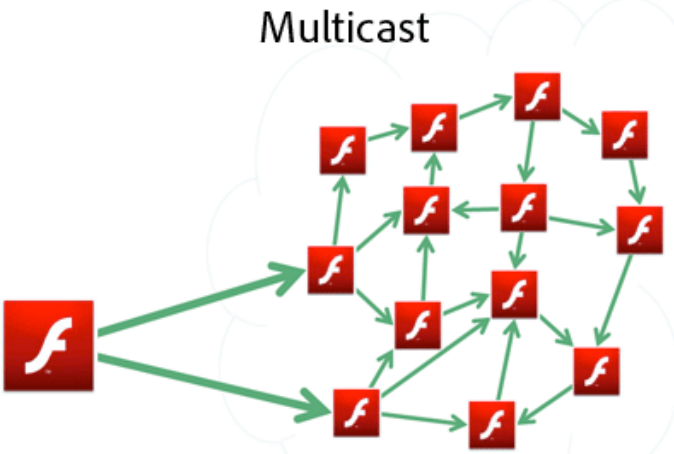

Figure 2. Structure of Flash Multicast

\section{B Packet distribution in Multicast}

Multicast in Flash is optimized to be efficient. It works the way that you don't upstream whole stream you received. It is a Pull/Push system. Pull ensures to deliver the data to the peers. Push optimizes the mesh, reduce latency and create multiple spanning trees.

1) Pull mechanism[12]

Once you start distributing stream, you send a map of blocks to the target receivers. They will search their neighbors to see who can source each block. Then they send request to the neighbors that have available blocks. Their neighbors will send the requested blocks to them but they can also refuse the request. If the request of receivers is unfulfilled, they can ask other neighbors.

\section{2) Push mechanism}

All clients can send map of what blocks they have to their neighbors who pick candidate sources for each sequence number slice and then send mask describing which sequence number to push. The push client periodically tests its neighbors to check for the lowest latency, so that it keeps the quickest source for each sequence number slice. Data is pushed immediately as it arrives for requested slice. For each slice, there is a limit to the number of push clients served.

Through combining two mechanisms above, multicast distribution is effective and optimized[14].

\section{Conclusion}

For Multicast in Flash, it uses NetStream class together with GroupSpecifier and NetConnection [5]. Multicast is the most complicated thing in Flash P2P implementation and it's very intelligent in optimizing the delivery, chunk distribution and mesh reforming.

\section{GRoup Audio ApPLICATION}

In this part, I will use a Group Audio application to illustrate how to build a Flash multicast streaming application with RTMFP. With this application, users can talk with each other through sending and receiving audio streams obtained from the microphone. The final result of this application is described in Figure 3.

\section{Choose User Name xlh1}

Neighbor count : 2 publish

$15: 38: 20: 281$ you have joined the group! 15:38:36:718 $x$ lh2 has joined the group!

$15: 43: 31: 703 \times$ lh 3 has joined the group!

$15: 45: 6: 921 \times \operatorname{lh} 2$ begins to publish!

$15: 45: 21: 500 \times 1$ lh 3 begins to publish!

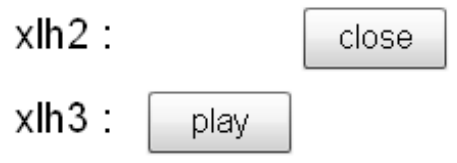

Figure 3. User interface of the Group Audio application

\section{A. Workflow}

This application is developed with Flash Builder 4 and deployed with a Tomcat Web project. Users just need to visit the URL address to enter the application.

1) Login and Configure

When you login, the connect button is not enabled. You should write your name down and the connect button will be enabled. Then click the connect button, the Flash Player will alert you to allow the peer assisted network to use your upload bandwidth. Just click allow button. Then you can see your neighbor count, some information of the group and the publish button.

2) Publish your Audio

Click the publish button, the Flash Player will alert you to allow accessing your camera and microphone. Just click allow, and your voice obtained through the microphone will 
be published to the group. At the same time, your neighbors will see that you begin to publish. You can also stop publishing by click the stop button.

3) Choose to play neighbors' Audio

Once your neighbor publishes his audio to the group, you will see his name and a play button following it. You can click play to receive his audio stream and click close button to stop receiving it.

\section{B. Implementation}

I build two projects with Flash Builder to implement this application. One is a Flex Library project called "LibP2P_Xlh" which manages the communication between you and your peers, so that there is no need to care about how the network communication is done in the business logic. It provides some useful functions:

- It dispatches events about what happens in the network.

- It provides the configuration parameters like RTMP server, developer key, application name and group name.

- It provides all the functions about the network communication. For example, when you click the "publish" button, a function called "startPublish" in this library will be executed.

The source tree of this project is shown in Figure 4.

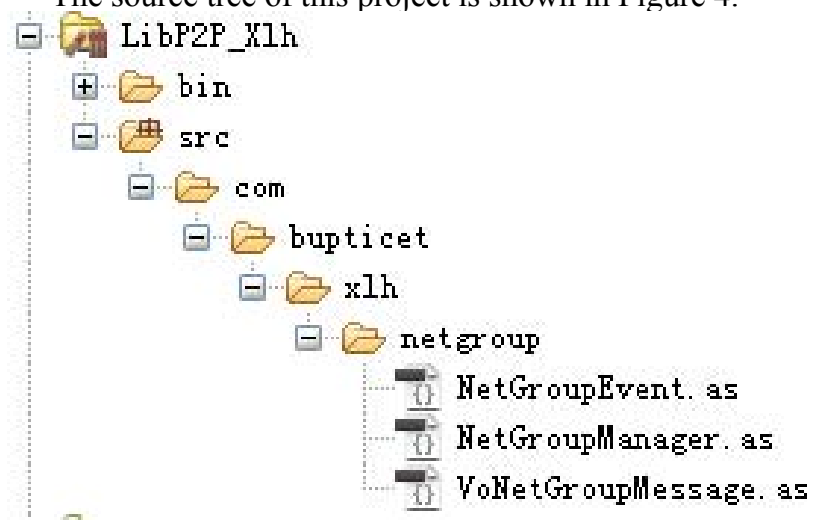

Figure 4. Flex Library project source tree

The other project is a Flex project which is called "P2PAudioTest". It is the main application providing the user interface and the business logic. The Flex Library project described above should be referred in this project and the "NetGroupManager" class is also instantiated in the main application in this project. The source tree of this project is shown in Figure 5.

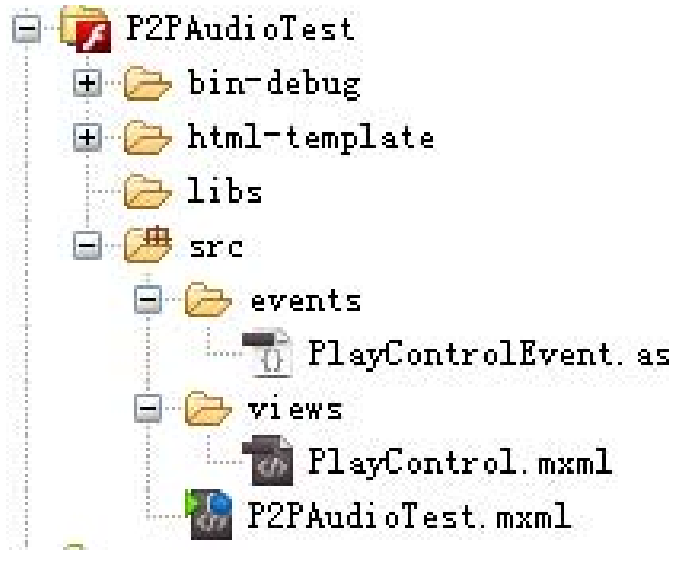

Figure 5. Flex project source tree

This application has been tested in the Local Area Network, and the voice quality is good. There is about one second latency when streaming the audio. It is applicable for medium scaled group chatting.

\section{CONCLUSION}

This paper introduces the Flash P2P feature shipped since Flash Payer 10 and the RTMFP which is built on User Datagram Protocol (UDP). This feature provides low latency and Data prioritization which make it very suitable for building Real-time RIA application. With its end-to-end media delivery feature, the load of media server will be dramatically reduced.

The deficiency of this feature is that it does not support recording the media streams, so you can not replay the audio and video of your peers if they are not online. Through combining Flash P2P and some media servers like FMS or Red5, this deficiency will be eliminated and the server load will be reduced significantly.

\section{ACKNOWLEDGMENT}

This paper is supported by the Key Project in the National Science \& Technology Pillar Program of China under grant No.2008BAH29B00 and the Fundamental Research Funds for the Central Universities under grant bupt2010pt10.

\section{REFERENCES}

[1] Wikipedia, "Speex", http://en.wikipedia.org/wiki/Speex, 2011-04-02

[2] Wikipedia, "Network address translation", http://en.wikipedia.org/ wiki/Network address translation, 2011-02-27.

[3] Jozsef Vass, "Cirrus service for developing end-to-end applications using RTMFP in Flash Player10", http://www.adobe.com/devnet/ flashplayer/articles/rtmfp_cirrus_app.html, 2011-02-22.

[4] Tom Krcha, "Directed Rounting Explained in Flash 10.1 P2P", http://www.flashrealtime.com/directed-routing-explained-flash-p2p/, 2010-07-04.

[5] Adobe System Inc. "BETA ActionScript ${ }^{\circledR}$ 3.0 Reference for the Adobe ${ }^{\circledR}$ Flash ${ }^{\circledR}$ Platform", http://help.adobe.com/en_US/Flash Platform/beta/reference/actionscript/3/, 2011-03-27 
[6] Tom Krcha, "Multicast Explained in Flash 10.1 P2P", http://www.flashrealtime.com/multicast-explained-flash-101-p2p/, 2010-07-01.

[7] Adobe System Inc. "What is application-level multicast?", http://www.adobe.com/products/ flashmediaserver/faq/, 2011.

[8] Tom Krcha, "Local Flash Peer-to-Peer Communication over LAN", http:/www.flashrealtime. com/local-flash-peer-to-peercommunication-over-lan-without-cirrus/, 2010-08-27.

[9] Adobe System Inc. "Use RTMFP for developing real-time collaboration applications", http://labs.adobe.com/technologies/cirrus/, 2011.

[10] Sebastien Portebois, "Building a P2P Network Vote System Using RTMFP”, http://active.tutsplus.com/tutorials/actionscript/building-ap2p-network-vote-system-using-rtmfp/, 2010-12-21.
[11] Wikipedia, "IP multicast", http://en.wikipedia.org/wiki/IP_multicast, 2010-03-20.

[12] Xiaojun Hei ; Yong Liu ; Ross, K.W. "IPTV over P2P streaming networks: the mesh-pull approach", Communications Magazine, IEEE Press, Feb. 2008, doi: 10.1109/MCOM.2008.4473088.

[13] Hayasake, M. ; Gamage, M. ; Miki, T. "Referential Loss Recovery for Streaming Audio using Application Level Multicast", Communications, 2005 Asia-Pacific Conference on, 5-5 Oct. 2005, doi: 10.1109/APCC.2005.1554061.

[14] Chia-Yi Liu ; Kuochen Wang ; Yi-Ling Hsieh, "Efficient push-pull based P2P multi-streaming using application level multicast ", Personal Indoor and Mobile Radio Communications (PIMRC), 2010 IEEE 21st International Symposium on, 26-30 Sept. 2010, doi: 10.1109/PIMRC.2010.5671773. 